\title{
Dynamic Subchannel and Power Allocation using Nash Bargaining Game for Cognitive Radio Networks with Imperfect PU Activity Sensing
}

\author{
Akram Baharlouei and Bijan Jabbari \\ Department of Electrical and Computer Engineering \\ George Mason University \\ Fairfax, VA 22030 \\ Email: \{abaharlo, bjabbari\}@gmu.edu
}

\begin{abstract}
This paper proposes a dynamic subchannel and power allocation scheme based on Nash Bargaining Game for an ad hoc network of Secondary Users (SU) coexisting opportunistically with a Primary base station. Each SU is equipped with an energy detector to sense the Primary User (PU) activity over $N$ OFDM sub-channels. We deploy Nash Bargaining Solution to model the power and subchannel allocation of the SUs over the temporarily available transmission opportunities. Simulation results show that the proposed dynamic power and subchannel assignment is simple, effective and fair. Moreover, the total throughput of the network is highly dependent on the accuracy level of the sensing information and the percentage of PU silence. An acceptable secondary network throughput is achievable if the probability of $\mathrm{PU}$ presence is limited to less than 0.4 .
\end{abstract}

Index Terms-Dynamic Resource Allocation, Nash Bargaining Solution, Water-filling, OFDM, Spectrum Sensing, Energy Detector.

\section{INTRODUCTION}

With ever-increasing demand for high data rate communications and wider bandwidth, spectrum scarcity is becoming an inevitable issue which reveals the inefficiency of the current static spectrum access techniques [1]. Cognitive radio technology as a potential platform to implement the Dynamic Spectrum Access (DSA) has been captured the interest of researchers in recent years [2]. Two major concerns in an opportunistic-access cognitive radio networks are spectrum sensing and spectrum allocation. A secondary user senses the spectrum of the Primary periodically, and uses it when it is idle, and switch to other unused bands when primary system is present. As we can see spectrum sensing is the first and critical step for a cognitive radio device to operate.

There are several detectors to sense the spectrum of a Primary User (PU) such as, matched filter, energy detector, and feature detection [3]. Feature detection is able to distinguish between the received signal energy and the noise energy but it requires long observation intervals of the received signal which leads to high computational complexity. Matched filter detector is the optimal option for the case of stationary Gaussian noise and when the primary signal is known. However, when the primary signal is unknown or complexity is an issue, matched filter and feature detection are ruled out, and energy detector appears to be the feasible choice.

The problem of dynamic spectrum allocation in OFDMA wireless networks has been widely studied in the literatures [4]-[7]. In [4] the dynamic channel assignment is modeled as a convex optimization problem to maximize the minimum of the sum of all users rate with respect to some constraint and a simple suboptimal algorithm is proposed. However, maximizing the worst user (max-min) rate is at the cost of sacrificing the users with better channel conditions which leads to a lower total rate. Another adaptive resource allocation scheme is proposed in [6] with taking proportional fairness into account by adding a constraint on rate requirement of each user. Nevertheless, Proportional fairness is a special case of NBS when the disagreement point is zero. Generally allocating subchannels in order to maximize the sum throughput of the network is a linear optimization problem with integer variables which could be solved using integer programming which is computationally complex. Hence, either suboptimum algorithms are proposed or the integer programming problem relaxed to a standard convex optimization.

By widespread use of wireless devices the available dynamic resource allocation schemes need to be even more flexible to be able to serve the unlicensed users while the primary users are in idle mode. This paper addresses this issue by using Nash Bargaining Solution (NBS) which provides optimality and fairness at the same time. NBS is a cooperative game where optimizes the multiplication of the objective function rather than the aggregate sum which results higher pay-off per user. In [8] a power control scheme is proposed based on NBS and KSBS for cognitive radio networks. [9] also proposes a resource allocation framework for an ad hoc network of secondary users using two game theoretic methods. However, in modeling the system there is no constrain for subchannel sharing among users and each subchannel can be allocated to several users which is not practical for OFDM networks and lowers the total throughput [10]. Moreover, solving and formulating the NB Game as a second approach is not complete. Another interesting approach for dynamic resource allocation using NBS approach is proposed in [7]. 
The problem is solved for two-user case in an iterative manner, but for the multiuser case the authors involve another game tool, called coalition games, which adds more iteration to the two-user case convergence. Furthermore, the convergence of the algorithm is not guaranteed.

The available literature applied NBS for only two user cases and the resulting optimization problem is not solved generally. This paper proposes an optimum and fair dynamic spectrum allocation schemes for the case of $K$ users.

In [11] the dynamic resource allocation in cognitive radio networks with Imperfect Channel Sensing is investigated and is solved using a discrete stochastic optimization method. However, the imperfectness is assumed for the channel gain information. Basically, the goal of spectrum sensing is to monitor the activity of the PU which is the case in our paper. An interesting joint cross-layer scheduling and spectrum sensing for cognitive radio networks is proposed in [12] based on what is called 'Raw Sensing Information' and the power and subchannel allocation is solved using primal-dual decomposition approach. Their underlay spectrum sharing method with assuming some acceptable interference level differs from our work where we adopt overlay method which does not allocate an OFDM sub-channel to more than one user simultaneously. Moreover, our problem solving method is based on NBS which gives a fair and simpler allocation scheme.

Following to our collaborative spectrum sensing work in [13], here, we use the sensing information of the energy detector of each SU to design an optimum power and subchannel allocation for SUs. First, we show that how the sensing bits of the PHY layer can affect the allocation process in MAC layer. Then, we propose a sub-optimum resource allocation algorithm in order to increase the network total throughput while maintaining fairness among users.

The remaining of this paper is organized as follows. Section II describes the system model. In section III the proposed method is elaborated. Simulation results are given in section IV and $\mathrm{V}$ concludes the paper.

\section{SySTEM MODEL}

Consider an adhoc secondary network with $K$ users competing over $N$ OFDM sub-channels of a Primary base-station. Each subchannel has a bandwidth of $w$. The rate for $i$-th user is expressed as:

$$
R_{i}=\sum_{n=1}^{N} w b_{i, n}
$$

where $b_{i, n}$ is the number of bits per symbol for the $i$-th user in subchannel $n$. Assuming $b_{i, n} \geq 2$ and BER $\leq 10^{-3}$, the following approximation will hold [14]:

$$
b_{i, n} \approx \log _{2}\left(1+c_{2} \gamma_{i, n} \ln \left(\frac{\mathrm{BER}_{i}}{c_{1}}\right)\right)
$$

where $c_{1}=0.2, c_{2}=1.5 . \mathrm{BER}_{i}$ is the $i$-th user bit error rate, and $\gamma_{i, n}$ is the SNR in subchannel $n$. Hence, the rate for user

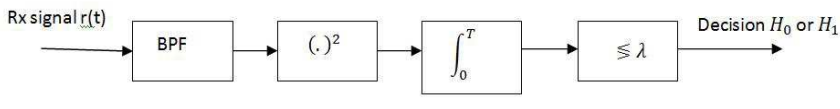

Fig. 1. Block Diagram of an Energy detector

$i$ can be formulated as:

$$
R_{i}=\sum_{n=1}^{N} c_{i, n} w \log _{2}\left(1+c_{3} \frac{p_{i, n} h_{i, n}^{2}}{\sigma_{i}^{2}}\right)
$$

where $c_{3}=c_{2} / \ln \left(c_{1} / \mathrm{BER}_{i}\right), \sigma_{i}^{2}$ is the noise power, and $h_{i, n}$ and $p_{i, n}$ are the channel gain and transmitted power of the $i$-th user on subchannel $n$ respectively. Moreover, the subchannel assignment coefficient $c_{i, n}$ is given as:

$$
c_{i, n}= \begin{cases}1, & \text { If subchannel } n \text { is allocated to user } i \\ 0, & \text { Otherwise. }\end{cases}
$$

On the other hand, we assume that each secondary user is equipped with an energy detector to sense the presence of PU on any of the $N$ subchannels [13]. The output of the detector $Y$, is compared with the threshold $\lambda$ to make a decision out of these two hypotheses(Figure 1 ):

$$
\begin{cases}Y \geq \lambda & H_{1} \\ Y<\lambda & H_{0}\end{cases}
$$

where $H_{1}$ indicates the presence of the PU and $H_{0}$ denotes that PU is inactive.

The probability that each SU correctly detects the presence of PU is $P_{d, i}$, and the probability that PU presence is falsely reported is $P_{f, i}$ :

$$
\begin{aligned}
& P_{d, i}=P\left\{Y>\lambda \mid H_{1}\right\} \\
& P_{f, i}=P\left\{Y>\lambda \mid H_{0}\right\}
\end{aligned}
$$

Based on the above definition the following four cases could occur (Figure 2):

1) PU is present and SU detects correctly

2) PU is present and SU detects falsely

3) PU is inactive and SU detects the same

4) PU is inactive and SU detects active

Taking into account the sensing information, we define the utility per SU as follows:

$$
\begin{aligned}
\bar{U}_{i} & =\sum_{n=1}^{N} \hat{H}_{i, n} r_{i, n} \\
& =\sum_{n=1}^{N} \hat{H}_{i, n} c_{i, n} w \log _{2}\left(1+c_{3} p_{i, n} G_{i, n}\right)
\end{aligned}
$$

where $\hat{H}_{i, n}$ is the sensing bit of the $i$-th user on subchannel $n$. $\hat{H}_{i, n}=0$ means that the subchannel is occupied by the PU whereas $\hat{H}_{i, n}=1$ indicates the availability of the the subchannel for SU use. 


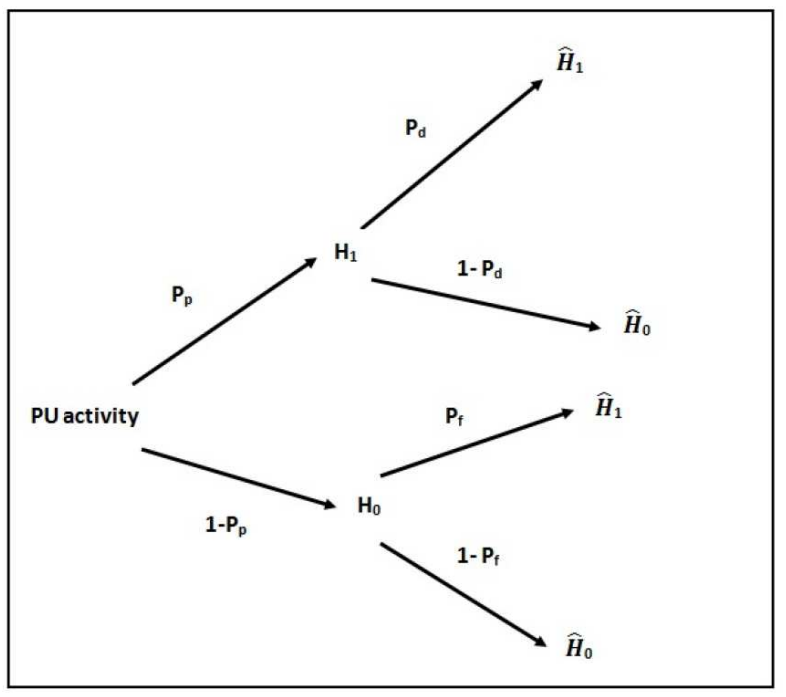

Fig. 2. Sequential graph for SU sensing

The problem is how to allocate the $N$ subchannels and subsequently the transmitted power among $K$ SUs so that the maximum throughput is achieved. In order to define the total throughput we take the product of the rates from Nash Bargaining game. We assume that channel state information of all users are known.

\section{A. Nash Bargaining Game}

Nash Bargaining game [15] is a class of cooperative games where there is a mutual agreement among users for cooperation in order to achieve a higher utility comparing to the non-cooperative case. Let's define $\mathbf{u}=\left(u_{1}, u_{2}, \ldots, u_{K}\right)$ as the utility vector. The minimum attainable utility for the users without cooperation is called the disagreement point, and is expressed as $\mathbf{u}^{\mathbf{0}}=\left(u_{1}^{0}, u_{2}^{0}, \ldots, u_{K}^{0}\right)$, and $U \in \mathbb{R}^{K}$ is the feasible utility set. Then, a $K$ player bargaining problem is a pair $\left\langle U, \mathbf{u}^{\mathbf{0}}\right\rangle$, where $U$ is a compact, bounded, and convex set, and there exists at least one utility pair $\left(u_{1}, u_{2}, \ldots, u_{K}\right) \in U$ such that $u_{1} \geq u_{1}^{0}, u_{2} \geq u_{2}^{0}, \ldots, u_{K} \geq u_{K}^{0}$. A bargaining solution is a function $\mathbf{u}^{*}=\left(u_{1}^{*}, u_{2}^{*}, \ldots, u_{K}^{*}\right)=F\left(U, \mathbf{u}^{\mathbf{0}}\right)$ that assigns a unique element of $U$ to the bargaining problem $\left\langle U, \mathbf{u}^{\mathbf{0}}\right\rangle$. This solution is given by:

$$
\mathbf{u}^{*}=\arg \max _{\mathbf{u} \in U, u_{1} \geq u_{1}^{0}, u_{2} \geq u_{2}^{0}, \ldots, u_{K} \geq u_{K}^{0}} \prod_{k=1}^{K}\left(\mathbf{u}_{\mathbf{k}}-\mathbf{u}_{\mathbf{k}}^{\mathbf{0}}\right)
$$

In order to model the resource allocation problem we set the utility function as follows:

$$
\begin{aligned}
\bar{U}_{i} & =E\left\{U_{i} \mid H_{i}\right\} \\
& =\sum_{n=1}^{N} E\left[\hat{H}_{i, n} \mid H_{i}\right] c_{i, n} w \log _{2}\left(1+c_{3} p_{i, n} G_{i, n}\right)
\end{aligned}
$$

On the other hand, from Figure 2 it is obvious that:

$$
\begin{aligned}
P\left\{\hat{H}_{i, n} \mid H_{i}\right\} & =\frac{\left(1-p_{p}\right)\left(1-p_{f}\right)}{\left(1-p_{p}\right)\left(1-p_{f}\right)+p_{p}\left(1-p_{d, i}\right)} \\
& =\beta_{i}
\end{aligned}
$$

where $P_{p}$ is the probability that $\mathrm{PU}$ is active on any of subchannels and $\beta_{i}$ indicates how accurately a SU can sense the presence of PU as is defined in [12] as well. For example, the case of $p_{d, i}=1$ results in $\beta_{i}=1$ which states that detection is done with \%100 certainty. Hence, (9) can be rewritten as:

$$
\bar{U}_{i}=\sum_{n=1}^{N} \beta_{i} c_{i, n} w \log _{2}\left(1+c_{3} p_{i, n} G_{i, n}\right)
$$

Therefore, $F\left(S,\left(\bar{U}_{1, \min }, \bar{U}_{2, \min }, \ldots, \bar{U}_{K, \min }\right)\right)$ is a bargaining problem where the set $S$ contains all the feasible rates, and its solution satisfies:

$$
\arg \max _{p_{1}, p_{2}, \ldots, p_{K}, c_{i, n}} \prod_{i=1}^{K}\left(\bar{U}_{i}-\bar{U}_{i, \min }\right)
$$

subject to:

$$
\begin{gathered}
C_{1}: \quad \sum_{i=1}^{N} P_{i} \leq P_{i, \max } \\
C_{2}: \quad \bar{U}_{i} \geq \bar{U}_{i, \min } \\
C_{3}: \quad c_{i} \in\{0,1\} \\
C_{4}: \quad \sum_{i=1}^{K} c_{i}=1 \\
C_{5}: \quad p_{i, n} \geq 0
\end{gathered}
$$

where $P_{i, \max }$ is the maximum power budget of the $i$-th user. Constraint $C_{4}$ ensures that each subchannel is assigned to one user only. This optimization problem is hard to solve as it is dealt with both continuous and binary variables. One approach is to relax the condition in $C_{4}$ by letting $c_{i}$ take values between $\left[\begin{array}{ll}0 & 1\end{array}\right]$. 


\section{Proposed ResourCe AlLOCATION SCHEME USing NASH BARGAINING GAME}

In order to solve (12), we first form the Lagrangian and apply the Karush-Kuhn-Tucker (KKT) conditions as follows:

$$
\begin{aligned}
L\left(p_{i, n}, c_{i, n}\right) & =\left(\sum_{n=1}^{N} \beta_{1} c_{1, n} w \log _{2}\left(1+c_{3} p_{1, n} G_{1, n}\right)-\bar{U}_{1, \min }\right) \\
& \ldots\left(\sum_{n=1}^{N} \beta_{i} c_{i, n} w \log _{2}\left(1+c_{3} p_{i, n} G_{i, n}\right)-\bar{U}_{1, \min }\right) \\
& -\sum_{i=1}^{K} \lambda_{i}\left(\sum_{n=1}^{N} p_{i, n}-P_{i, \max }\right) \\
& -\sum_{n=1}^{N} \mu_{n}\left(\sum_{i=1}^{K} c_{i, n}-1\right) \\
& -\sum_{i=1}^{K} \sum_{n=1}^{N} \nu_{i, n} p_{i, n}
\end{aligned}
$$

Hence, the KKT conditions are:

$$
\begin{aligned}
\frac{\partial L\left(p_{i, n}, p_{i, n}\right)}{\partial p_{i, n}} & =0 \\
\frac{\partial L\left(p_{i, n}, c_{i, n}\right)}{\partial p_{i, n}} & =0 \\
\lambda_{i}, \nu_{i, n}, \mu_{n} & \geq 0 \\
\sum_{i=1}^{K} \lambda_{i}\left(\sum_{n=1}^{N} p_{i, n}-P_{i, \max }\right) & =0 \\
\sum_{n=1}^{N} \mu_{n}\left(\sum_{i=1}^{K} c_{i, n}-1\right) & =0
\end{aligned}
$$

where $G_{i, n}=\frac{h_{i, n}^{2}}{\sigma^{2}}$.

From (14) we get the following power allocation equation:

$$
p_{i, n}=\frac{\prod_{j=1, j \neq i}^{K}\left(\bar{U}_{j}-\bar{U}_{j, m i n}\right)}{\lambda_{i}} \beta_{i} c_{i, n} w-\frac{1}{c_{3} G_{i, n}}
$$

Assuming subchannel $n$ is assigned to user $i$, i.e. $c_{i, n}=1$, (19) has the familiar face of a water filling equation with slightly changes in the water level. Hence, more power will be allocated to the subchannels with higher gains. On the other hand, (15) and (18) yields to:

$$
\frac{\beta_{1} \log 2\left(1+c_{3} p_{1, n} G_{1, n}\right)}{\left(\bar{U}_{1}-\bar{U}_{1, \min }\right)}=\cdots=\frac{\beta_{i} \log 2\left(1+c_{3} p_{i, n} G_{i, n}\right)}{\left(\bar{U}_{K}-\bar{U}_{K, \text { min }}\right)}
$$

As it is seen, getting a closed form solution for $p_{i, n}$ and $c_{i, n}$ from (19) and (20) is an NP-hard problem. However, it casts light on the shape of the optimum solution. Looking at $c_{i, n}$ each fraction can be interpreted as the rate of each user in one subchannel to the total rate of all subchannels assigned to that user which illustrates the ratio of the rate in one subchannel to the total rate should be the same for all users. This clue asserts the fairness of the optimal solution and ,also, gives us a metric for subchannel allocation.

\section{A. The Proposed Sub-optimum Power and Sub-channel Allo- cation Algorithm}

As it is seen in previous section, Nash product terms are hard to handle especially with the large number of users and subchannels. Hence, we break the problem into two subproblems. First, we assume that the subchannels are allocated,i.e. $c_{i, n}$ is known, from (19) and (17) we get the following familiar water filling equation:

$$
p_{i, n}=\frac{P_{i, \max }+\frac{1}{c 3} \sum_{i=1}^{N} \frac{1}{G_{i, n}}}{N}-\frac{1}{c 3 G_{i, n}}
$$

Then, we propose the following algorithm for subchannel allocation by modifying the algorithm in [4] as follows:

1) Initialization:

a) Set $\bar{U}_{i}=0, \Omega_{i}=\emptyset$ for all $i=1,2, \ldots, K$ and $A=\{1,2, \ldots, N\}$.

2) for $\mathrm{i}=1$ to $\mathrm{K}$,

a) Find $n$ satisfying $\left|G_{i, n}\right| \geq\left|G_{i, k}\right|$ for all $k \in A$.

b) Let $\Omega_{i}=\Omega_{i} \cup\{n\}, A=A-\{n\}$ and update $\bar{U}_{i}$ according to (11).

3) While $A \neq \emptyset$,

a) find $i$ satisfying $\frac{G_{i, n}}{\sum_{j \in \Omega_{i}} G_{i, j}} \leq \frac{G_{m, n}}{\sum_{j \in \Omega_{m}} G_{m, j}}$ for all $m, 1 \leq m \leq K . \Omega_{i}$ and $\Omega_{m}$ are the subchannels allocated to user $i$ and $m$ respectively;

b) For the found $i$, find $n$ satisfying $\left|G_{i, n}\right| \geq\left|G_{i, k}\right|$ for all $k \in A$

c) For the found $i$ and $n$, let $\Omega_{i}=\Omega_{i} \cup\{n\}, A=A-\{n\}$ and update $R_{i}$ according to (11).

4) Water fill power based on (21) for each $\Omega_{i}$, $i=1,2, \ldots, K$.

\section{Simulation Results}

In this section we testify the performance of the proposed subchannel and power allocation approach under different sensing parameters. It is assumed that the sub-channel gains are i.i.d. random variables with Rayleigh distribution. Noise spectral density is $N_{0}=-110 \mathrm{~dB}$ and is the same for all $K$ users. The total available bandwidth is $w=3.2 \mathrm{MHz}$, and the maximum allowable power per user is $0.3 \mathrm{~W}$. Without loss of generality we set $\bar{U}_{i, \min }=0$. First, we consider the case that $\mathrm{PU}$ is completely silent which results in $\beta_{i}=1$. Hence, the problem reduces to allocating $N$ subchannels among $K$ users without requiring any sensing information. In this case we are able to compare the proposed algorithm with the available methods(max-min and sum-max) for the non-cognitive case as it is seen in Figure 3. It shows the allocated rate per user per subchannel for $N=16$ subchannels and $K=6$ users with gray bars for the proposed algorithm, black bars for the maxsum rate and white bars for the max-min case. Let's consider the two extreme cases first. It is obvious that the first user has the worst channel condition. As we see the max-sum algorithm 


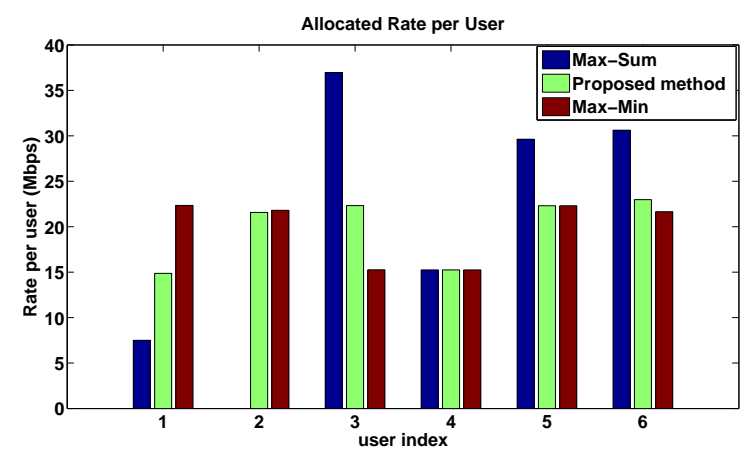

Fig. 3. A comparison of the allocated rate per user for the Max-Min, MaxSum, and the proposed algorithm $(\mathrm{N}=16, \mathrm{~K}=6)$

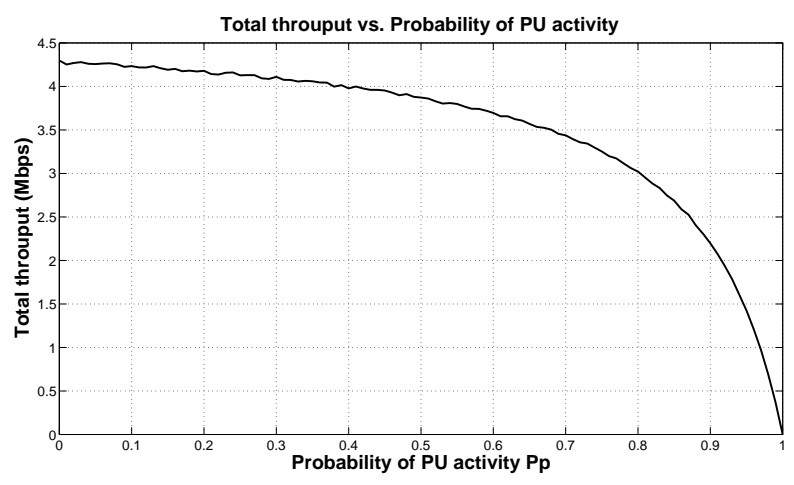

Fig. 4. Total throughput vs. the probability of PU activity $(K=12, N=32)$

does not allocate any subchannel to this user since it lowers the total rate while the max-min approach treats this user almost the same as other users at the cost of reducing the total rate of the network. The proposed algorithm, however, aiming at balancing the optimality and the fairness, assigns the lowest rate to this user. Let's switch to the second user which is the best user with the highest channel gain. The max-sum allocates the highest rate to this user but the max-min assigns a rate even less than the worst case user which results in the underutilization of the second user good channel conditions. The proposed algorithm keeps the middle position. The same analysis applies for the rest of users which are between the two first extreme cases.

Figure 4 illustrates the total throughput of the $K=6$ users with $N=32$ subchannels versus the probability of the PU activity. We observe that for the values of $P_{p}<0.4$ the throughput does not drop that much. However, when $P_{p}$ passes 0.5 the total throughput drops rapidly which can give us a threshold on where the co-existence of a PU and SUs are beneficial.

In Figure 5 we assess the total throughput of the Secondary network under different values of $\beta$. We assume that all SUs are using an energy detector with the same parameters, therefore, the index $i$ of $\beta$ could be dropped. The very bottom plot is correspond to the case of $\beta=0$ which could be mapped to the case that $\mathrm{PU}$ is active all the time $\left(P_{p}=1\right)$ or the case

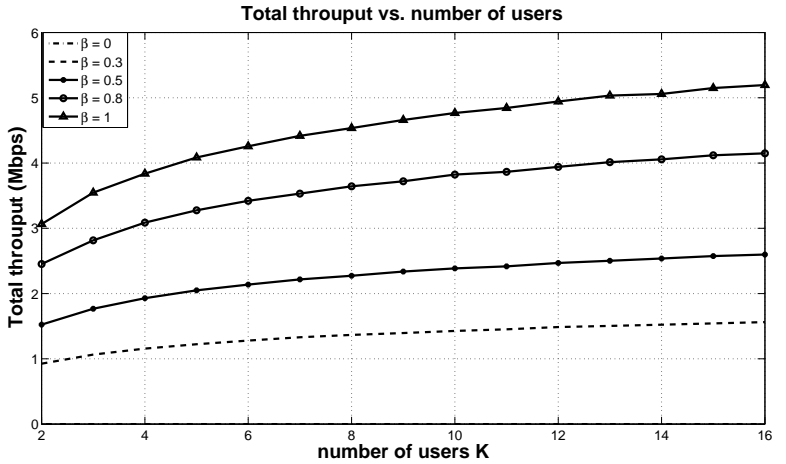

Fig. 5. Total throughput vs. number of users $(\mathrm{N}=32)$

that $P_{f}=0$. In either case there would be no transmission opportunity for the SUs and no throughput will be achieved. As $\beta$ increases the total throughput is growing accordingly which indicates that the more accurately the SUs sense the higher throughput is obtained. The maximum throughput is for the case of $\beta=1$ which means either SUs are sensing perfectly $P_{d}=1$ or PU is completely silent $P_{p}=0$.

\section{CONCLUSION}

An effective dynamic subchannel and power allocation algorithm is proposed based on Nash bargaining Solution for an ad hoc network of secondary users with imperfectly sensing PU activity. Comparing to the max-sum approach which totally ignores the users with weak channel conditions and the max-min scheme which maximizes the worst user rate at the cost of scarifying the good users, the proposed algorithm balances these two extreme cases by weighting each user according to its total channel gain. Moreover, it is shown that the total throughput of the secondary network is highly related to how accurately the sensing process is performing and how frequently the PU is present. The higher detection probability is desirable in order to take the best advantage of the temporarily available PU subchannels. Furthermore, a PU with more than $\% 40$ activity lowers the SU throughput sharply which could be an indicator for the SUs that the PU is not worth sensing.

\section{REFERENCES}

[1] B. Jabbari, R. Pickholtz,and M. Norton, "Dynamic Spectrum Access and Management," vol. 17, pp. 6-15, Aug. 2010.

[2] J. Mitola and G. Q. Maguire, "Cognitive radio: Making software radios more personal," vol. 6, 1999.

[3] D. Cabric, M. S. Mishra, and R. W. Broderson, "Implementation issues in spectrum sensing for cognitive radios," 2004.

[4] W. Rhee, and J. Cioffi, "Increase in Capacity of Multiuser OFDM System using Dynamic Subchannel Allocation," in Proc. IEEE VTC, 2000.

[5] W. Yu, and J. M. Cioffi, "FDMA Capacity of Gaussian Multiple-Access Channels with ISI," IEEE Trans. Commun., vol. 50, no. 1, 2002.

[6] Z. Shen, J. G. Andrews, and B. L. Evans, "Adaptive Resource Allocation in Multiuser OFDM Systems with Proportional Rate Constraints," IEEE Trans. Wireless Commun., vol. 4, no. 6, 2005.

[7] Z. Han, Z. Ji,and K. J. R. Liu, "Fair Multiuser Channel Allocation for OFDMA Networks Using Nash Bargaining Solutions and Coalitions," IEEE Trans. Commun., vol. 53, no. 8, 2005. 
[8] Chun- Yang, Jian-Dong Li, and Zhi Tian, "Optimal Power Control for Cognitive Radio Networks Under Coupled Interference Constraints: A Cooperative Game-Theoretic Perspective," IEEE Trans. Vehic. Technol., vol. 59, no. 4, 2010.

[9] A. Attar, M. R. Nakhai, and A. H. Aghvami, "Cognitive Radio Game for Secondary Spectrum Access Problem," IEEE Trans. Wireless Commun., vol. 8, no. 4, pp. 2121-2131, 2009.

[10] J. Jang, and K. B. Lee, "Transmit Power Adaptation for Multiuser OFDM Systems," IEEE J. Select. Areas Commun., vol. 21, pp. 171$178,2003$.

[11] R. Xie, F. R. Yu, and H. Ji, "Dynamic Resource Allocation for Heterogeneous Services in Cognitive Radio Networks with Imperfect Channel Sensing," IEEE Trans. Vehic. Technol., vol. 61, no. 2, 2012.

[12] R. Wang, V. K. N. Lau,L. Lv and B. Chen, "Joint Cross-Layer Scheduling and Spectrum Sensing for OFDMA Cognitive Radio Systems," IEEE Trans. Wireless Commun., vol. 8, no. 5, 2009.

[13] A. Baharlouei and B. Jabbari, "A stackelberg game spectrum sensing scheme in cooperative cognitive radio networks," IEEE Wireless Communications and Networking Conference, 2012.

[14] S. T. Chung, and A. Goldsmith, "Degree of Freedom in Adaptive Modulation: a unified view," IEEE Trans. Commun., vol. 49, no. 9, 2001.

[15] J. Nash Jr, "The Bargaining Problem," Econometrica, vol. 18, no. 2, pp. $155-162,1950$. 